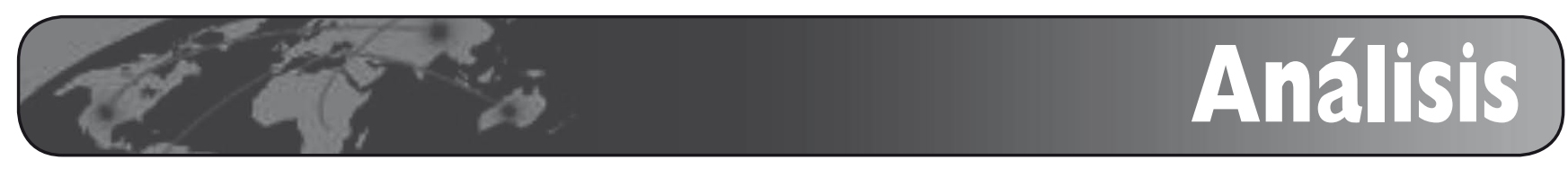

\title{
Webs de ciudades. El caso de Bcn.cat
}

\author{
Por Marc Garriga-Portolà
}

Resumen: La historia de la web de la ciudad de Barcelona-Bcn. cat- se remonta a 14 años atrás. En este tiempo ha pasado de ser una web pequeña, estática y con poco contenido a ser una de las webs municipales más importantes, con 100.000 visitas al día y un millón de páginas servidas diariamente. En este artículo se presentan datos relacionados con dicha web y con la penetración y uso de internet en la ciudad de Barcelona.

Palabras clave: Barcelona, Barcelona 2.0, Internet, Estadísticas, Web, Web 2.0, Análisis web.

\section{Title: City webs. The Bcn.cat case}

Abstract: The history of the municipal web of the city of Barcelona -Bcn.cat-goes back to 14 years ago. In this time has passed to be a

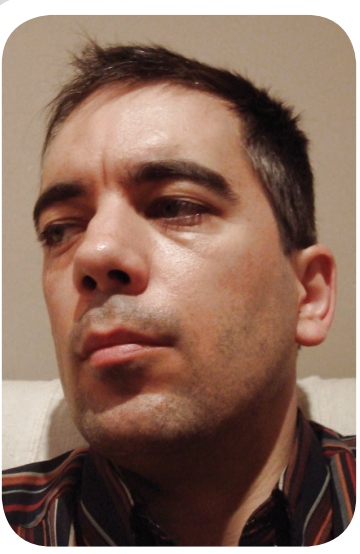
Marc Garriga Portolà es ingeniero en informática por la Universitat Politècnica de Catalunya y licenciado en investigación y técnicas de mercado (marketing) por la Universitat Oberta de Catalunya. Es el responsable de indicadores web del Ayuntamiento de Barcelona y desde hace 3 años trabaja para el departamento técnico de internet $y$ nuevos canales de dicho Ayuntamiento. small web, static and with little content to be one of the more important municipal webs with 100,000 visits per day and a million pages served daily. This article shows data related to this web and with the Internet penetration and use in the city of Barcelona.

Keywords: Barcelona, Barcelona 2.0, Internet, Statistics, Web, Web 2.0, Web Analysis.

Garriga-Portolà, Marc. "Webs de ciudades. El caso de Bcn.cat". El profesional de la información, 2009, enero-febrero, v. 18 , n. 1 , pp. $63-66$.

DOI: 10.3145/epi.2009.ene.08

\section{Introducción}

DESDE LA ADMINISTRACIÓN PÚBLICA LOCAL se ha ido tomando conciencia de que internet permite llegar de forma más rápida y sencilla a los ciudadanos para ofrecerles la información que necesitan.

Las webs de ciudades, a cargo de sus ayuntamientos, introducen continuas mejoras tecnológicas y aprovechan este medio para acercarse a la gente. En este artículo se analiza el caso de Barcelona.

\section{Qué es Bcn.cat}

Bcn.cat es el dominio principal de la web de la ciudad de Barcelona. Está integrada por unas 200 subwebs, 80.000 páginas estáticas, un gestor de contenidos corporativo y una amplia amalgama de tecnologías web.
La primera versión se creó en mayo de 1995, ya hace casi 14 años, una eternidad en el mundo de internet. En sus comienzos era puramente informativa y estática, y fue creada con ayudas de la Unión Europea.

\section{"Bcn.cat recibe diariamente 100.000 visitas y sirve cerca de un millón de páginas"}

En la actualidad es uno de los canales de comunicación más importantes con que cuenta el Ayuntamiento de Barcelona. Se ha integrado plenamente en la gestión municipal, como demuestra el hecho de que 1,2 millones de tramitaciones $^{1}$ se han realizado desde ella en
2008, mejorando la comodidad de los ciudadanos al realizar trámites y, lo que es más importante, aumentando la eficiencia de los servicios municipales.

Hay que tener presente que toda la web Bcn.cat, según los datos más recientes $^{2}$ de 2008, recibe una media cercana a las 100.000 visitas diarias y sirve casi un millón de páginas al día.

\section{Datos del uso de internet por los ciudadanos}

Es una realidad evidente que nuestra sociedad se está digitalizando y conectando a internet cada vez más. De hecho ya se habla de la "sociedad red" y de todas las implicaciones que se derivan de esta sociedad conectada.

En el caso de la ciudad de Barcelona podemos expresar esta digi- 
talización de la sociedad con cifras. Según datos municipales ${ }^{3}$, el $57 \%$ de los ciudadanos utiliza asiduamente el ordenador, el 55\% acostumbra a utilizar internet y un $52 \%$ tiene conexión a internet desde casa; la mayoría (más de un $88 \%$ de estos últimos) tiene una conexión de calidad: por ADSL o por cable.

La periodicidad con la que se conectan a internet es muy variable. El $70 \%$ lo hace cada día, el 26\% semanalmente y un $4 \%$ con una periodicidad superior. Respecto al lugar de conexión, el $81 \%$ se conecta desde casa, un $37 \%$ desde el trabajo, un $8 \%$ desde un locutorio, un $5 \%$ desde la escuela y más de un $6 \%$ desde un cibercafé o un centro cultural (hay que tener en cuenta que es habitual conectarse desde varios sitios, de ahí que el resultado de la suma sea superior al 100\%).

\section{"Los ciudadanos de Barcelona valoran su web municipal con una puntuación de 7,2"}

Los motivos principales para la utilización de internet son: búsqueda de información (63\% de los que se conectan), chatear (22\%), trabajo $(20 \%)$ y recepción y envío de correos (14\%). En menor porcentaje, entre el 5 y el $10 \%$, tenemos la navegación sin un propósito concreto (9\%), estudiar (7\%), bajarse música o películas $(6 \%)$ y realizar reservas de viajes (6\%); por debajo del $5 \%$ el uso de internet es para jugar (2\%), consultar la prensa $(2 \%)$, realizar transacciones bancarias $(1,5 \%)$ y otros. Como en el caso del lugar de conexión, la suma da un resultado superior al $100 \%$ ya que es habitual, y lógico, conectarse por varios motivos.

Los ciudadanos de Barcelona valoran su web municipal Bcn.cat

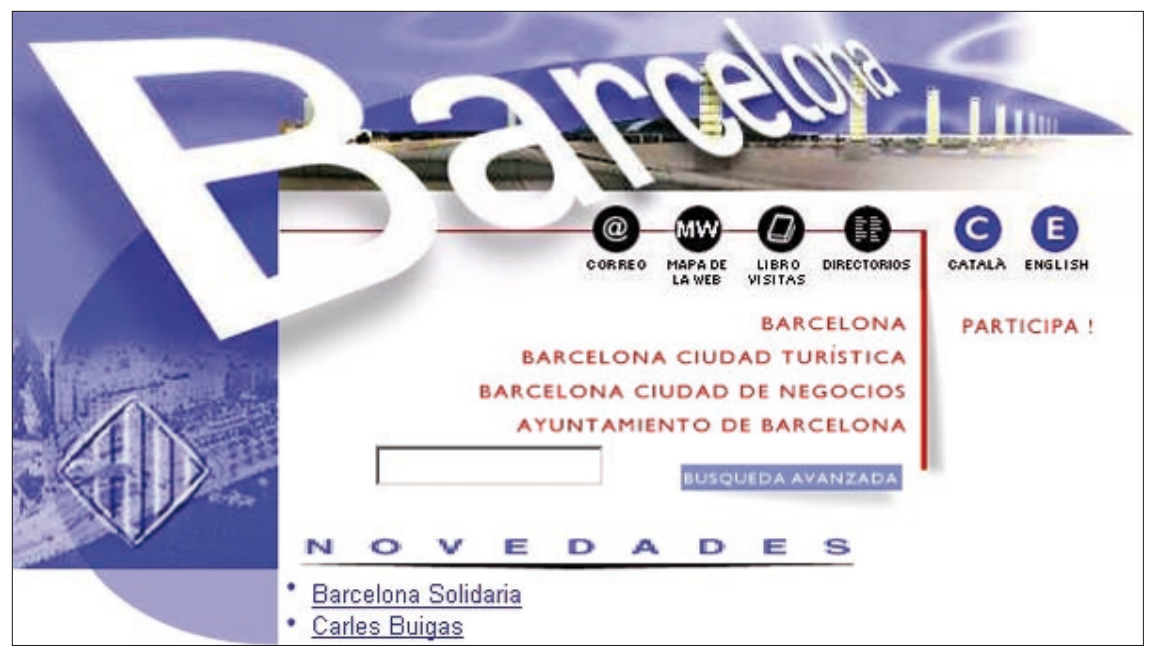

Figura 1. BCN año 1999

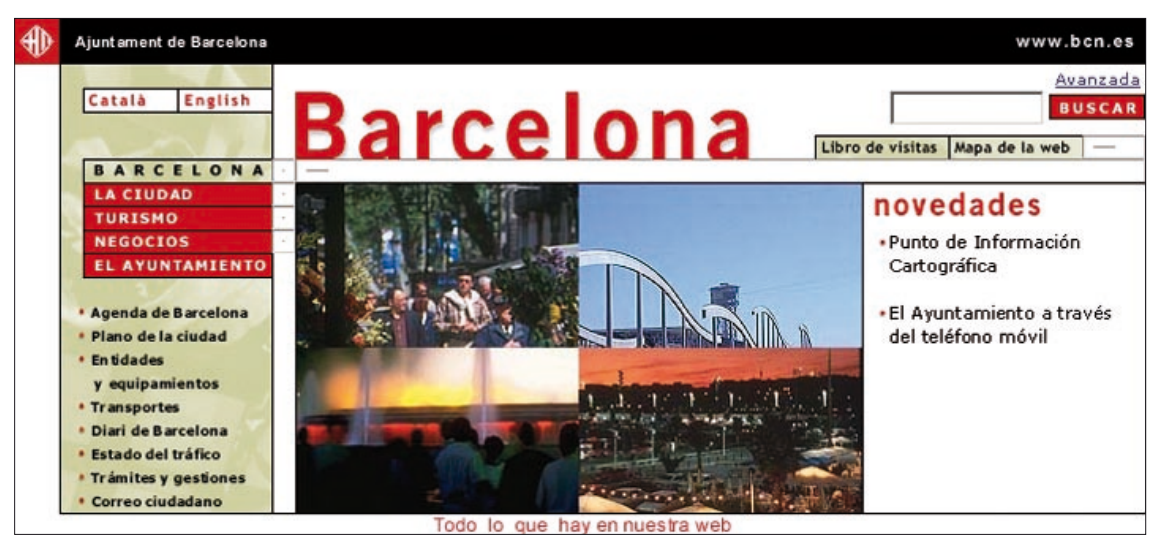

Figura 2. BCN año 2000

con una puntuación de notable, un 7,2 sobre 10 . Este nivel de satisfacción se ha mantenido estable desde 2004, cuando se llevó a cabo por primera vez la encuesta de satisfacción.

De todas maneras hay que decir que el resto de los canales municipales de información también están bien valorados: el mejor es el servicio de información en el móvil (barcelona.mobi) que recibe una puntuación de 7,9; las oficinas de atención al ciudadano y el teléfono del civismo (900 226 226) reciben un 7,2; y el teléfono de información municipal (010) un 7,1.

\section{Datos analíticos de Bcn.cat}

Si profundizamos más en los datos que presenta la web Bcn.cat, podemos centrarnos en los técnicos y en los de "negocio".
La duración de las visitas es alta: la media (según datos hasta octubre de $2008^{4}$ ), es de casi 11 minutos, con una mediana de más de 2 minutos (recordemos que la mediana es el valor que divide en dos partes el total de elementos de una serie, en este caso, las duraciones de todas las visitas recibidas). Son cifras respetables teniendo en cuenta la cantidad de visitas que se reciben y el tipo de web de que se trata (por ejemplo no es un sitio con un volumen importante de documentos largos).

"En los últimos 10 años Bcn.cat ha recibido 184 millones de visitas y ha servido 1.715 millones de páginas" 
Más datos técnicos ${ }^{5}$ demuestran la "salud" de la web: está disponible más del 99,5\% del tiempo (una mejora de $1 \%$ respecto al año anterior). Sólo un $1,85 \%$ de las peticiones son errores, y la inmensa mayoría de ellos son provocados por peticiones a páginas inexistentes.

Si se analizan los datos que en una web comercial serían del "negocio" y que aquí simplemente son los temas, se puede definir el enfoque de Bcn.cat y lo que más interesa a los ciudadanos.

Según las estadísticas del período 1 de enero a 31 de octubre de 2008, el apartado con más visitas fue el de movilidad y seguridad, con un $27 \%$ del total de visitas. Esto se debe básicamente a que el callejero -el servicio con más aceptación- forma parte de este tema. Sin embargo los intereses son muy heterogéneos, es decir, parece haber muchas áreas atractivas para los ciudadanos que les llevan a visitar la web municipal de Barcelona. En la tabla 1 se listan las 20 materias más consultadas.

La tabla 1 contiene los temas con más visitas a la web, sin detenerse en el detalle de la "calidad". Por ejemplo la mayoría de las visitas al callejero son muy cortas, en cambio las consultas a directorios

\begin{tabular}{|l|c|}
\hline \multicolumn{1}{|c|}{ Temática } & $\begin{array}{c}\text { Porcentaje } \\
\text { de visitas } \\
\text { (\%) }\end{array}$ \\
\hline Movilidad y seguridad (incluye el callejero) & 27,3 \\
\hline Información cultural & 10,4 \\
\hline $\begin{array}{l}\text { Información institucional (administración pública, } \\
\text { ayuntamiento, alcalde) }\end{array}$ & 6,2 \\
\hline Comercio, innovación y promoción económica & 5,3 \\
\hline Turismo & 5,2 \\
\hline Información territorial (incluye información de distritos y barrios) & 4,7 \\
\hline Educación & 3,4 \\
\hline Documentación & 3,2 \\
\hline Deportes, ocio y tiempo libre & 2,9 \\
\hline Directorios y catálogos & 2,8 \\
\hline Información de actualidad (incluye las noticias) & 2,8 \\
\hline Servicios sociales, derechos civiles y juventud & 2,7 \\
\hline Urbanismo y vivienda & 2,4 \\
\hline Fiestas y tradiciones & 2,2 \\
\hline Medio ambiente & 1,4 \\
\hline Participación y espacios participativos & 0,9 \\
\hline Gestión del conocimiento y TIC & 0,4 \\
\hline Secciones relacionadas con eventos especiales & 0,4 \\
\hline Juegos y espacios emocionales & 0,2 \\
\hline Otros temas & 15,3 \\
\hline
\end{tabular}

Tabla 1: Visitas a la web Bcn.cat según los temas buscados

y catálogos o a noticias, en general son más largas.

Cabe destacar que un $27,8 \%$ de las visitas son de origen internacional muy repartido. Sólo 6 países -a parte de España- tienen un porcentaje de visitas a la web Bcn.cat superior al 1\%(tabla 2).

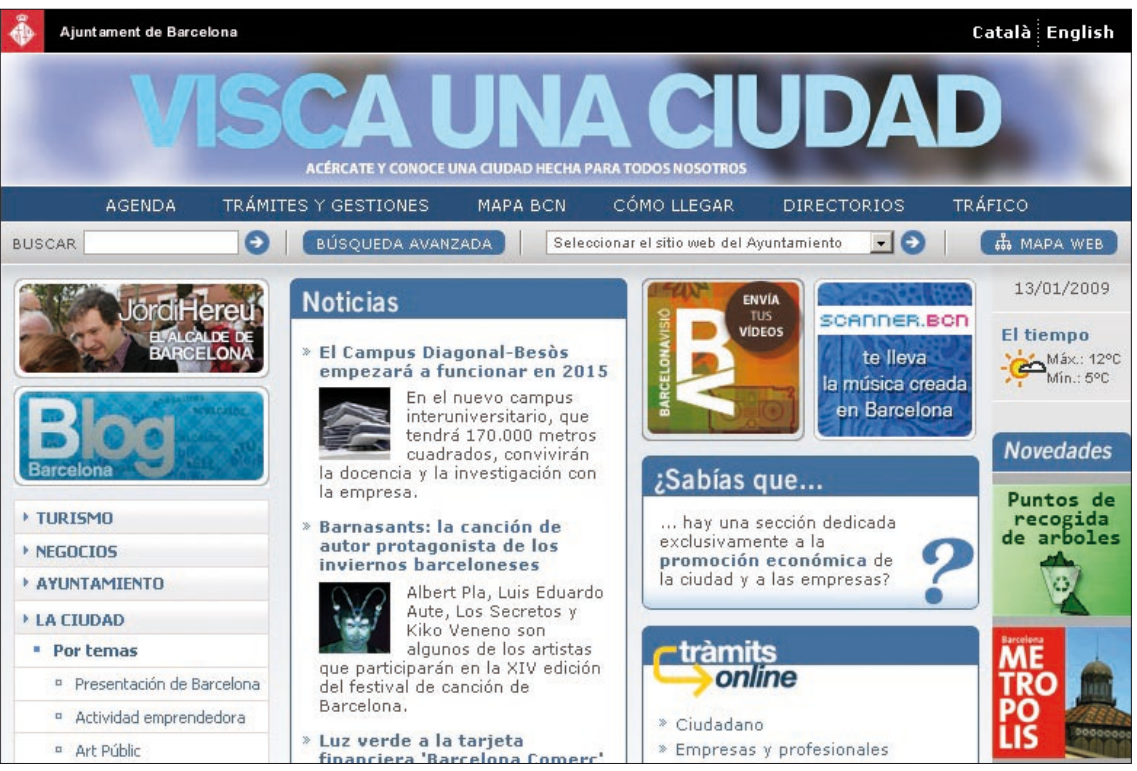

Figura 3. BCN año 2009

\begin{tabular}{|l|c|}
\hline \multicolumn{1}{|c|}{ País } & $\begin{array}{c}\text { Porcentaje } \\
\text { de visitas } \\
(\%)\end{array}$ \\
\hline España & 72,2 \\
\hline Estados Unidos & 5,1 \\
\hline Reino Unido & 2,7 \\
\hline Francia & 1,5 \\
\hline Alemania & 1,5 \\
\hline Uruguay & 1,4 \\
\hline México & 1,0 \\
\hline Otros países & 14,6 \\
\hline
\end{tabular}

Tabla 2: Países que proporcionan como mínimo un $1 \%$ de visitas a la web Bcn.cat

Respecto a los sitios que tienen enlaces a la web Bcn.cat y le proporcionan visitas (los llamados referrers), hay que destacar que más de 4 de cada 10 visitas son de tráfico directo (usuarios que han escrito directamente el dominio $B c n$. cat en su navegador, pues es fácil de recordar y de escribir, o que han llegado a Bcn.cat desde su listado de favoritos). Una de cada 3 proviene del buscador Google, que es quien proporciona más visitas (tabla 3 ). 


\begin{tabular}{|l|c|}
\hline \multicolumn{1}{|c|}{ Referrer } & $\begin{array}{c}\text { Porcentaje } \\
\text { de visitas } \\
\text { (\%) }\end{array}$ \\
\hline Tráfico directo & 43,0 \\
\hline Google & 29,1 \\
\hline $\begin{array}{l}\text { Versión online de El } \\
\text { periódico de Cata- } \\
\text { lunya }\end{array}$ & 14,0 \\
\hline $\begin{array}{l}\text { Bcn.cat (referrers } \\
\text { internos) }\end{array}$ & 5,0 \\
\hline Yahoo! & 0,6 \\
\hline Live.com & 0,5 \\
\hline Resto de referrers & 14,6 \\
\hline
\end{tabular}

Tabla 3: Referrers que proporcionan como mínimo un $0,5 \%$ de las visitas a la web Bcn.cat

\section{Evolución histórica}

Debido al carácter minoritario que inicialmente tuvo, habitual en las webs de aquella época, se carece de estadísticas hasta mayo de 1997.

El primer año completo del que se dispone de datos es 1998: medio millón de visitas y casi cinco millones de páginas servidas. Diez años después estas cifras se consiguen en tan sólo 5 días.

Queda evidente el extraordinario crecimiento experimentado en poco tiempo, lo que ha provocado una continua necesidad de mejora de los medios técnicos asociados, así como de los recursos de planificación.

Para los aficionados a las cifras, un dato de interés: desde el 26 de mayo de 1997 -primer día con estadísticas- hasta el 31 de diciembre de 2008, la web Bcn.cat ha recibido más de 184 millones de visitas y ha servido casi 1.715 millones de páginas.

\section{Futuro de Bcn.cat}

Como se acostumbra ahora, hay que estar siempre en permanente "fase beta", para seguir avanzando y adaptarse tanto a los cambios tecnológicos como a las necesidades de la gente. El modelo actual está en proceso de reflexión, una meditación sobre qué web municipal queremos que tenga la ciudad de Barcelona en el futuro. Para ello se tienen en cuenta los indicadores obtenidos con los análisis de la web.

La filosofía que conlleva el fenómeno Web 2.0 y, en general la so-

"Se está en un proceso de reflexión para elaborar un nuevo modelo de web" ciedad red, es un factor más que nos empuja hacia una reformulación de los objetivos.

Al mismo tiempo se está implantando un nuevo modelo de gestión municipal que pretende mejorar la calidad de los servicios y potenciar la proximidad con los ciudadanos. Se ha bautizado como Barcelona 2.0, y en él las TICs serán pieza clave.

\section{Notas}

1. Ayuntamiento de Barcelona. Depto. técnico de internet y nuevos canales. Avance del resumen de las tramitaciones realizadas en la web Bcn.cat en 2008. Barcelona, enero 2009.

2. Ayuntamiento de Barcelona. Depto. técnico de internet y nuevos canales. Avance del resumen de las estadísticas de la web Bcn.cat en 2008. Barcelona, enero 2009.

3. Ayuntamiento de Barcelona. Dirección de servicios de estudio y evaluación. Encuesta semestral de sistemas de información y atención a Barcelona (Essiab). Barcelona, julio 2008.

4. Ayuntamiento de Barcelona. Depto. técnico de internet y nuevos canales. Resumen de las estadísticas de la web Bcn.cat de octubre 2008. Barcelona, noviembre 2008

Marc Garriga-Portolà, Depto. Técnico de Internet y Nuevos Canales, Dirección de e-Administración, Ayuntamiento de Barcelona. mgarrigap@gmail.com

\section{¿Sabías que ZPl tiene blog?}

\section{Se llama "Zona de notas"}

\section{http: //wWw.elprofesionaldelainformacion.com/notas/}

\section{En él puedes seguir toda la} actualidad y noticias sobre "El profesional de la información": novedades, comentarios sobre los contenidos, call for papers, reseñas de actividades, noticias sobre EPI en otros medios, etc.

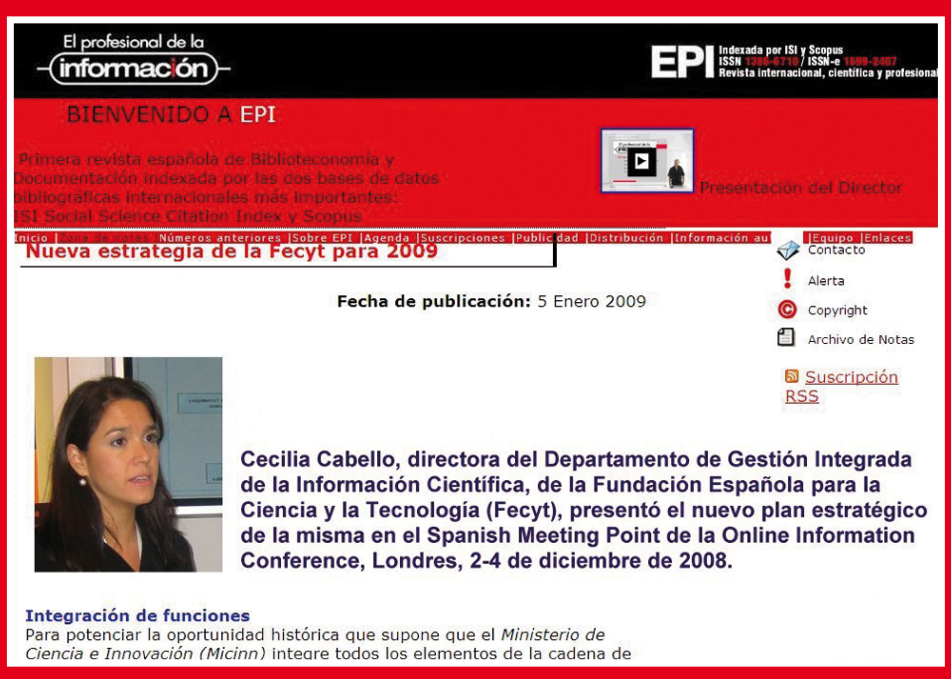

